\title{
AUTOMATED ON-DISC TOTAL RNA EXTRACTION FROM WHOLE BLOOD TOWARDS POINT-OF-CARE FOR EARLY STAGE DIAGNOSTICS
}

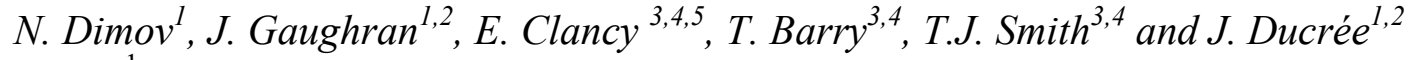 \\ ${ }^{1}$ Biomedical Diagnostics Institute, Dublin City University, IRELAND \\ ${ }^{2}$ School of Physical Sciences, Dublin City University, IRELAND \\ ${ }^{3}$ Microbiology, School of Natural Science, National University of Ireland, Galway, IRELAND \\ ${ }^{4}$ Molecular Diagnostics Research Group, National University of Ireland, Galway, IRELAND \\ ${ }^{5}$ Biomedical Diagnostics Institute Programme, National University of Ireland, Galway, IRELAND
}

\begin{abstract}
We present a novel integrated, centrifugo-pneumatic micro-homogenizer (" $\mu$ Homogenizer") for automated sample preparation and total RNA extraction from whole blood. Using a Trizol based protocol, this novel $\mu$ Homogenizer efficiently lyses whole blood spiked with E. coli, retains the organic-mixed fraction and yields the aqueous phase with the total RNA content. By the interplay of microfluidic design and a protocol of rotational frequencies, we concatenate (and parallelize) a sequence of five subsequent liquid handling operations that complete in less than 10 minutes. A comparison of the total nucleotide content yields similar performance as conventional, essentially manual off-disc sample preparation methods.
\end{abstract}

\section{KEYWORDS}

On-disc diagnostics, three-phase extraction, RNA, spiked blood, bacterial infections.

\section{INTRODUCTION}

Bacterial infections are amongst the leading causes of death globally. Early-stage diagnosis of the causative agent of infection is key for improved healthcare [1]. Infections are presently diagnosed by time-consuming bacteria culture or faster PCR which both require cumbersome sample preparation. Utilizing standard microfabrication methods for rapid prototyping and advances in microfluidics, we successfully integrate ondisc a protocol for total RNA extraction from $E$. coli spiked blood samples.

Whilst Trizol is routinely used to isolate RNA from biological samples, its application in microfluidic on-chip sample preparation has not been thoroughly investigated. One possible explanation for this is the highly corrosive nature of the reagent and the challenge of finding appropriate resistant materials. Another is the technical challenge of on-chip phase separation.

These challenges are addressed in this paper on automated centrifugo-pneumatic mixing and purification on-disc. Our long-term objective is to develop a rapid, integrated and fully automated sample-to-answer platform with the here presented " $\mu$ Homogenizer" [2] at its pivot. In the present work, the stand-alone mixing functionality [3] is significantly advanced by an entirely rotationally controlled loading of on-disc stored reagents as well as integrated retention of a metered supernatant volume and finally the collection of the purified, aqueous phase extract.

\section{ON-DISC MICROFLUIDIC DESIGN}

\section{Concept of the centrifugo-pneumatic mixing}

This type of mixing is based on the balance between the centrifugally induced pressure head $\Delta p$ and the pressure of air that is trapped in the side arms [2] of the $\mu$ Homogenizer (Figure 1).

$$
\Delta p=\rho \omega^{2} \Delta r \bar{r}
$$

Here, $\rho$ is the average density of the liquids; $\omega$ is the angular velocity of the disc; $\Delta r$ is the radial extent of the liquid; $\bar{r}$ is the average radial position of liquid from the center of rotation, defined by the interfaces of liquid / air in the mixing chamber and liquid / compressed air in the side arm. Using Boyle's law the balance can be written as:

$$
\rho \omega^{2} \Delta r \bar{r}=P_{A T M} \frac{V_{D}}{V_{M C}+V_{S A}}
$$

$P_{A T M}$ is the ambient pressure as the fluid is loaded; $V_{D}$ is the displacement of liquid from the mixing chamber to the side arms; the volume of liquid in the mixing chamber is $V_{M C}$; and in the side arms is $V_{S A}$. This equation permits the calculation of the angular velocity $\omega$ at which the maximal displacement is reached.

\section{Siphon valve and restriction on angular velocity}

The siphon valve prevents the liquid from flowing over to the retention chamber; however, it also restricts the angular velocity $\omega$. Behind the working principle of the siphon valve lies another equilibrium; between the capillary force inside the channel and the centrifugal force. These two forces point in opposite directions and determine the position of the meniscus with relation to the angular velocity $\omega$ of the disc.

Above a certain threshold $(20 \mathrm{~Hz}$ in the current device) the liquid is kept below the crest point of the siphon. Going below this critical velocity triggers elution of the liquid: the organic phase in the retention and the aqueous phase in the collection chamber. Therefore, the angular velocity $\omega$ of the disc exceed the critical frequency of the siphon. The siphon valve restricts the lower limit of $\omega$ to a specific interval. To improve mixing in the $\mu$ Homogenizer, a nozzle constriction is milled at the bottom of the mixing chamber and the side arms, creating turbulence each time liquid is displaced. As a result mixing within the $\mu$ Homogenizer can be controlled by actuating the angular velocity. 


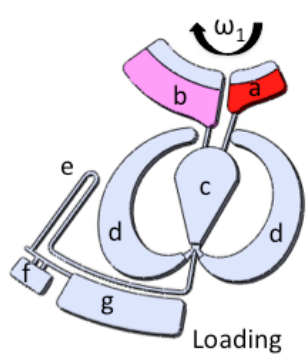

a) blood reservoir

b) Trizol BD ${ }^{\otimes}$ reservoir

c) mixing chamber

d) side arms

e) siphon valve

f) retention chamber

g) collection chamber
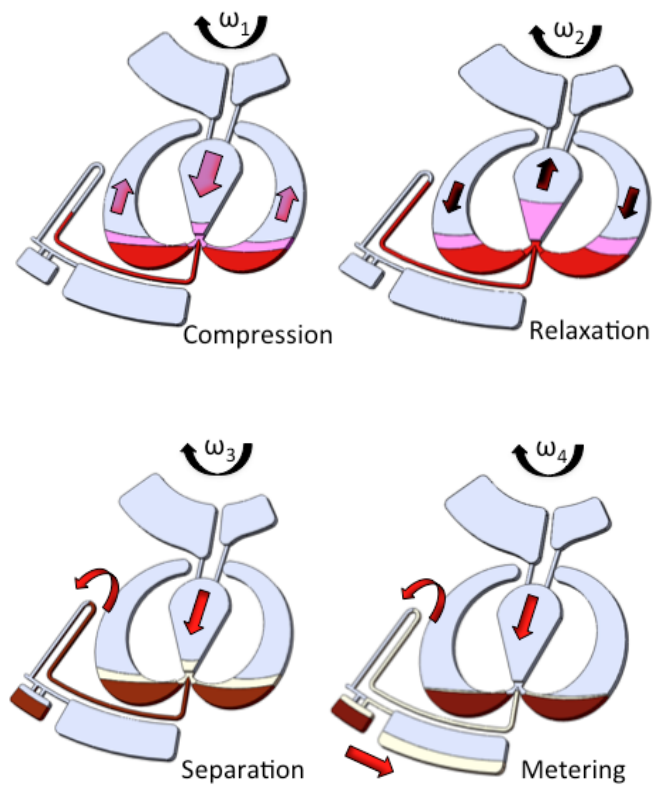

Figure 1: Components of the $\mu$ Homogenizer and the five functional regimes at different angular velocities $\omega_{i}$ of the disc. The fluid transport through the system is demonstrated in each functional mode. Shown are the released and compressed states of liquids at the mixing module of the device.

\section{EXPERIMENTAL SETUP}

\section{Disc milling and assembly}

The device was designed using Solid Works 2011 (Dassault Systems, US). All four structures and through wholes were milled (MDX-40A, Roland, DG, UK) in Zeonor (Zeon, Tokyo, Japan) disc $(d=120 \mathrm{~mm}$, $h=1.2 \mathrm{~mm}$ ). After the milling the discs were sonicated at $50^{\circ} \mathrm{C}$ for $30 \mathrm{~min}$ in Micro 90 ( $2 \%$ aq.) solution followed by another $30 \mathrm{~min}$ sonication in MilliQ water. Next, the discs were dried at $80^{\circ} \mathrm{C}$ for $40 \mathrm{~min}$. Using a contoured pressure sensitive adhesive (PSA), the structures were sealed with a second Zeonor disc $(d=120 \mathrm{~mm}$, $h=0.6 \mathrm{~mm}$ ). This three-layer assembly (Figure 2) was then run through a roller-press until completely bonded.

Spiking blood with $E$. coli and priming of the disc

To demonstrate proof of concept, the device was used to extraction total RNA from whole blood spiked with $E$. coli cells at a concentration of $3.75 \times 10^{4}$ colony forming units (CFUs) per $\mathrm{mL}$ (based on a plate count). For this, whole blood was spiked off-chip with $10 \mu \mathrm{L}$, $5 \mu \mathrm{L}$ or $2.5 \mu \mathrm{L}$ of bacteria to reach a total volume of
$25 \mu \mathrm{L}$. Figure 1 illustrates a 5-step protocol. The prepared sample was loaded by pipette into the $\mu$ Homogenizer blood reservoir (Figure 1, a); separately, $80 \mu \mathrm{L}$ of Trizol-BD was added into the adjacent reservoir (b).

\section{Total RNA extraction on-disc}

These liquids were then centrifugally loaded into the mixing chamber (c) with the two side arms (d) at angular velocity $100 \mathrm{~Hz}\left(\omega_{1}\right)$. At the 3-way intersection a nozzleconstriction enhances the turbulent mixing between the liquids, which are pneumatically driven by the expanding gas volumes in the side arms as the frequency is reduced to $50 \mathrm{~Hz}\left(\omega_{2}\right)$. Above a spin rate of $20 \mathrm{~Hz}\left(\omega_{3}\right)$ the siphon valve (e) prevents the liquid from leaving the compression / relaxation zone (c). The metered volume of mixed fraction, i.e. phenol and cell debris, was separated from the aqueous phase in a retention chamber (f) which features two parallel ducts bypassing the microchannel. Even in its filled state, this chamber continuously separates cell debris from the main flow due to the significant centrifugal force at $12.5 \mathrm{~Hz}\left(\omega_{4}\right)$, while the aqueous phase flows from the mixing chamber, through the siphon valve into the collection chamber $(\mathrm{g})$.

\section{Bench-top RNA quantification}

The aqueous phase from the collection chamber $(\mathrm{g})$ was analyzed for the presence of RNA by UV absorbance at 260nm (NanoDrop 2000, Thermo Scientific, US) for each of the spiked samples.

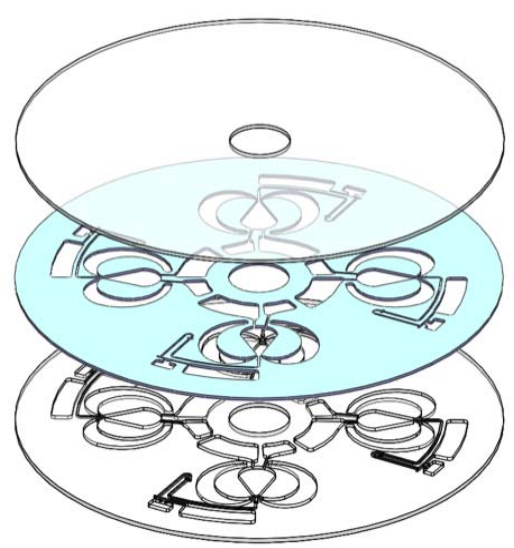

Figure 2: Multi-layer assembly of the $\mu$ Homogenizer. Consisting of two Zeonor discs bonded by a layer of pressure sensitive adhesive (PSA).

\section{RESULTS AND DISCUSSION}

Currently the automated sample homogenization requires 30 cycles to completely homogenize the $E$. coli spiked blood sample. The sample is in contact with the TRI-BD for 10 minutes. To reduce sample preparation time, the multi-layer disc assembly (Figure 2) can accommodate four parallel homogenization structures. This permits the simultaneous and independent processing of up to four samples on a single use disc. Keeping the time of contact constant; we measured the RNA quantity and purity from the different samples (Figure 3) and compared it to the standard "In tube" bench-top protocol. 
Table 1. Average amount of total RNA from whole blood samples processed using the $\mu$ Homogenizer and conventional bench-top methods. Duplicated samples were measured (Nano Drop 2000) and each value was normalized to the initially extracted volume of its aqueous phase.

\begin{tabular}{|l|c|c|c|c|}
\hline \multirow{2}{*}{$\begin{array}{l}\text { Method of sample } \\
\text { preparation }\end{array}$} & \multicolumn{4}{|c|}{ Volume of $\boldsymbol{E}$. coli used for spiking whole blood } \\
\cline { 2 - 5 } & $10 \mu \mathrm{L}$ & $5 \mu \mathrm{L}$ & $2.5 \mu \mathrm{L}$ & $0 \mu \mathrm{L}$ \\
\hline$\mu$ Homogenizer & $5.3 \mathrm{ng}_{\mu} \mathrm{L}^{-1}$ & $3.9 \mathrm{ng} \mu \mathrm{L}^{-1}$ & $3.5 \mathrm{ng} \mu \mathrm{L}^{-1}$ & $3.4 \mathrm{ng}_{\mu} \mathrm{L}^{-1}$ \\
\hline In tube & $3.4 \mathrm{ng} \mu \mathrm{L}^{-1}$ & $1.2 \mathrm{ng}^{-1}$ & $0.9 \mathrm{ng} \mu \mathrm{L}^{-1}$ & $1.2 \mathrm{ng} \mu \mathrm{L}^{-1}$ \\
\hline
\end{tabular}

A summary of total RNA recovered from the $\mu$ Homogenizer is presented in Table 1 . The total RNA extracted from the spiked samples is composed of nucleic acids from the blood and the bacteria. Despite the limitations of using absorbance at $260 \mathrm{~nm}$ to quantify total RNA, such as the inability to differentiate DNA from RNA, we evaluated the total amount of the extracted nucleic acids from the samples. All $\mu$ Homogenizer samples $(n=8)$ consistently recovered higher amounts of total RNA when compared to the bench-top protocol.
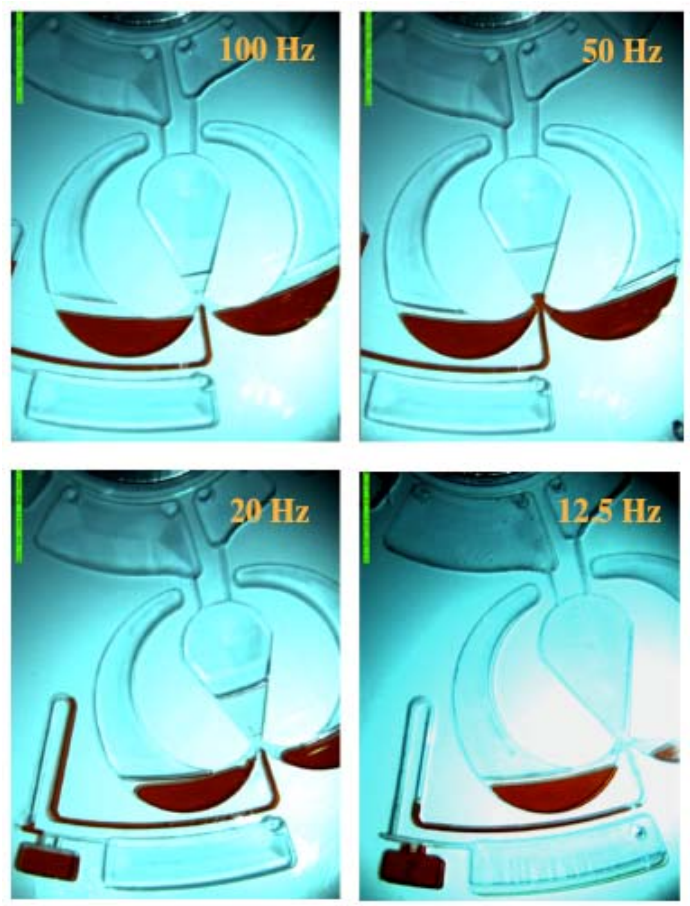

Figure 3: Sample preparation. Whole blood and TRI are mixed in less than 10 minutes by swiftly alternating the angular velocity $\omega$. After the siphon valve primes, a metered volume of non-reacted TRI from the capillary and debris as well as unmixed dead volume is retained in a small side chamber before purified total RNA in aqueous is collected.

The spectrophotometry ratios (Figure 4) carry information about the purity of the samples and the performance of the system. The 260/280 ratio of pure DNA is 1.8 whilst pure RNA has a $260 / 280$ ratio of 2 . Figure 3 shows that the organic phase, containing the DNA, is separated in the retention chamber and only the aqueous phase flows into the collection chamber. We compare two representative curves showing slightly higher ratios of the on-disc processed samples in comparison to the bench-top extraction. Although it does not explain the 260/280 ratios lower than 2, this gives sound evidence for an efficient extraction in the retention chamber, i.e. in the same chamber where most of the organic phase is retained.

The extraction protocol, which we implemented for both the bench-top and on-disc experiments, did not include any phase separation reagents like 4-bromoanisole or chloroform. In authors opinion during the intensive mixing an emulsion is formed. Resulting in trace amounts of phenol that travel in the main stream with the aqueous phase into the collection chamber. According to the technical specification of the Nano Drop these contamination is likely to be the cause of variations in the 260/280 ratios. Further studies and addition of phase separation agents are necessary to minimize the contamination.

Our second ratio (260/230) with values less than 1 indicates residual guanidine contamination originating from the guanidine dissolved in the aqueous phase. Abnormal absorbance at $230 \mathrm{~nm}$ is related to other contaminations, i.e. phenol and guanidine. Overall spectra display no traces of Trizol-BD with its characteristic peak at $220 \mathrm{~nm}$. Essentially for downstream analysis further sample purification is required because of the guanidine denaturing effect on proteins.
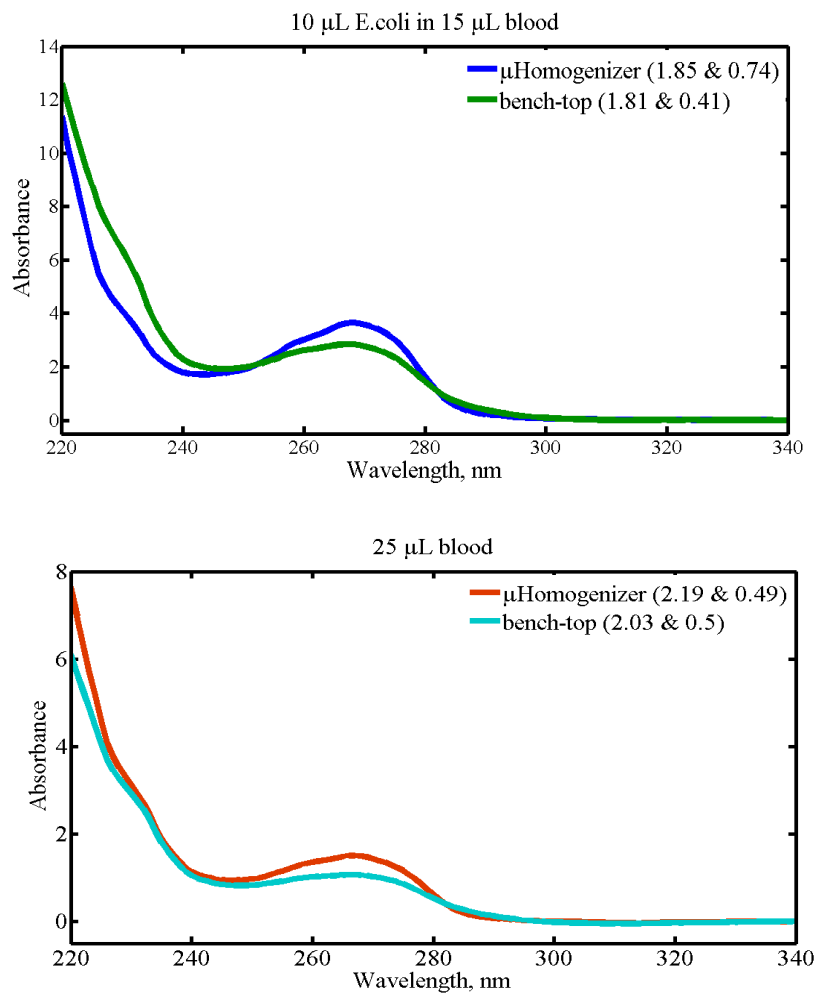

Figure 4: The spectra obtained from the analysis of the aqueous phase. 260/280 \& 260/230 ratios are given (inset) 


\section{CONCLUSIONS}

In summary, we presented on-disc centrifugopneumatic device for rapid E. coli spiked blood sample homogenization and extraction. Furthermore we compared extraction with the $\mu$ Homogenizer to its tubebased counterpart, showing similar if not better efficiency of separation on-disc. Future work will include optimization of the RNA extraction protocol towards minimum contamination by adding phase separation reagents. The $\mu$ Homogenizer will be a key constituent of a full-fledged sample-to-answer diagnostic for RNA detection in whole blood.

\section{ACKNOWLEDGEMENTS}

This work was supported by the Science Foundation of Ireland under Grant No 10/CE/B1821.

\section{REFERENCES}

[1] M. Mahalanabis, H. Al-Muayad, M. Kulinski, D. Altman, C. Klapperich, "Cell Lysis and DNA Extraction of Gram-positive and Gram-negative Bacteria from Whole Blood in a Disposable Microfluidic Chip", Lab on a Chip, vol. 9, pp. 28112817, 2009.

[2] A. Linares, R. Gorkin III, B. Glynn, N. Godino, N. Miller, M. Kerin, T. Barry, T. Smith and J. Ducrée, "Purification of miRNA from Whole Blood by Chemical Lysis and Phase Separation in a Centrifugo-pneumatic Micro-homogenizer", Proc. $\mu T A S$ Conference, Seattle, October 2-6, 2011, pp. 1460- 1462.

[3] R. Gorkin III, J. Park, J. Siegrist, M. Amasia, B. Lee, J. Park, J. Kim, H. Kim, M. Madou, Y. Cho", Centrifugal Microfluidics for Biomedical Applications" Lab on a Chip, vol. 10, pp. 1758$1773,2010$.

\section{CONTACT}

*N. Dimov, tel: +353-700-5889;

nikolay.dimov@dcu.ie 